\title{
Nesfatin-1: An Anorexigenic Peptid
}

\author{
Gulin Ozturk Ozkan (Corresponding author) \\ Health Science Faculty, Nutrition and Dietetics Department, \\ Istanbul Medeniyet University, Cevizli Campus, Kartal, Istanbul, Turkey \\ E-mail: gulin.ozturkozkan@medeniyet.edu.tr
}

\begin{abstract}
Nesfatin-1 was identified as a potent anorexigenic peptide. Nesfatin-1 is expressed in the brain and peripheral tissues. Precursor of nesfatin-1 is nucleobindin-2 (NUCB2). This polypeptide is involved in the control of energy balance by food intake. Peripheral nesfatin- 1 can potentially affect central nervous food intake regulation either via direct access to the brain. Circulating nesfatin-1 acts in the brain to influence food consumption, peripheral delivery of nesfatin-1 may reduce food intake and body weight. Nesfatin-1 appears to contribute to the regulation of food intake by directly and/or indirectly affecting the integration of input and output signals between gut and brain. Nesfatin-1 is association with obesity and glucose homeostasis. Plasma levels of nesfatin-1 can correlate with body mass index, insulin resistance, fasting blood glucose, and fasting insulin levels, as well as with body weight and fat mass, suggesting that nesfatin-1, especially fat-derived nesfatin-1, may play an important rol in human metabolism and food intake. In addition to, lipid accumulation may be reduced by nesfatin-1in cultured hepatocytes. In conclusion, it can be said that nesfatin-1 is not only associated with food intake but also with obesity, insulin resistance and type 2 diabetes.
\end{abstract}

Keywords: Nesfatin-1, anorexigenic peptid, food intake

DOI: $10.7176 / \mathrm{JSTR} / 5-2-27$

\section{Introduction}

Nesfatin-1 is an 82-amino acid hormone that derived from the nucleobindin-2 (NUCB2) precursor protein (Su et al., 2010; Yosten, 2013). Nesfatin-1 is a cleavage product of nucleobindin 2 (NUCB2), the sequence of which is highly conserved from fish to mammals (Dore et al., 2017). In quest of novel appetite regulating molecules, in 2006, Oh-I and coworkers re-discovered NEFA/nucleobindin2 (NUCB2), a peroxisome proliferator $\gamma$ receptor (PPARG)-activated gene in immortalized cell lines and later in the hypothalamus of rodents (Oh et al., 2006). Its gene product NUCB2, a 396 amino acid (AA) peptide, which was originally described as a secreted protein of unknown function, possesses a number of putative cleavage sites, suggesting further processing (Oh et al., 2006). The N-terminal fragment, named nesfatin1 , was subsequently identified in rat cerebrospinal fluid (CSF) and the hypothalamic paraventricular nucleus (PVN), and it was found to dose-dependently reduce food intake when administered intracerebroventricularly (i.c.v.) in rodents (Dore et al., 2017).

NUCB2 mRNA is mainly expressed in gastric mucosa and white adipose tissue and also, but to a minor extent, in other peripheral organs, e.g. pancreas and testis (Dore et al., 2017). Within the central nervous system (CNS), NUCB2 mRNA is present, for example, in the hypothalamus and brainstem (Oh et al., 2006). The product of the NUCB2 gene is a 420 AA peptide consisting of a 24 AA signal peptide and 396 AA peptide termed NUCB2. The latter possesses putative cleaving sites for protein convertases (PC) 1/3 and PC2, suggesting further processing into three alleged fragments: nesfatin-1 (AA 1-82), nesfatin-2 (AA 85-163) and nesfatin-3 (AA 166-396) (Oh et al., 2006). NUCB2 is co-localized with these enzymes in the cytoplasm, suggesting that processing of NUCB2 may take place physiologically (Dore et al., 2017). In the brain, nesfatin-1/NUCB2 mRNA or immunoreactivity has been detected in various hypotalamic nuclei, including feeding centers such as the arcuate nucleus, paravetricular nucleus, lateral hypotalamic area, dorsomedial hypotalalmus, and ventromedial hypotalalmus. In the periphery, nesfatin-1 is produce by white adipose tissue, particulary by subcutaneous fat depots, and by pancreatic beta cells. Additionally, nesfatin-1 has been detected in endocrine cells of the gastric mucosa (Yosten, 2013).

\section{Nesfatin-1 in the control of food intake}

Nesfatin-1 is a bioactive polypeptide expressed both in the brain and peripheral tissues and involved in the control of energy balance by reducing food intake (Dore et al., 2017). The first physiological function 
described for nesfatin-1 was the reduction of food intake in the dark phase after central injection in rats. In addition, a reduction in weight gain and fat depots most likely related to the anorexigenic action was also observed. In this context, all of the evidence suggests a physiological role for nesfatin-1 as a negative regulator of food intake (Folgueira et al., 2014). Central administration of nesfatin-1 significantly increases energy expenditure (Dore et al., 2017). Potential source of plasma nesfatin-1 include the adipose tissue, the endocrin cells of the gastric mucosa, and intestines, and the endocrine pancreas, particularly the pancreatic beta cells (Yosten, 2013).

Nesfatin-1 and its precursor NUCB2 possess anorexigenic properties. The application of the two other putative cleavage products of NUCB2, nesfatin-2 and/or nesfatin-3 was not effective. Endogenous levels of NUCB2/nesfatin-1 play a role in regulating feeding behavior (Dore et al., 2017).

Altough it is unclear whether circulating nesfatin-1 acts in the brain to influence food consumption, peripheral delivery of nesfatin-1 may reduce food intake and body weight. Two separate mechanisms for this effect have been proposed. First of all, it is known that nesfatin-1 is capable of crossing the bloodbrain barrier via a nonsaturable mechanism, and thus circulating nesfatin-1 could potantially act directly on feding centers in the brain following diffusion out of the plasma into the parenchyma. However, nesfatin-1 can activate vagal afferent neurons, suggesting an indirect modulation of central feeding circuits. Regardless of the mechanism, nesfatin- 1 is produced by adipocytes, it is reasonable to propose that nesfatin-1, like leptin, is an adipokine that relays metabolic information from the periphery to the brain (Yosten, 2013).

It has been recently reported by two separate groups that nesfatin- 1 can cross the blood-brain barrier without saturation. Both endogenous and peripherally administered exogenous nesfatin-1 can reach the brain, and inhibit feeding behavior (Shimizu et al., 2009).

Nesfatin-1 could fail to suppress light-phase food intake. When given intranasally, nesfatin-1 may reduce cumulative food intake, similar to leptin, most likely by directly accessing the brain. As Nucb2 mRNA and protein are expressed in the periphery, the effects of its putative cleavage product nesfatin- 1 in suppressing food intake after peripheral administration were also studied. Intraperitoneal (i.p.) acute administration of nesfatin-1 midsegment decreased food intake in both lean and $\mathrm{db} / \mathrm{db}$ leptin-resistant obese mice, thus suggesting that also peripheral nesfatin-1 induces anorexia in a leptin-independent manner. Peripheral nesfatin-1 can potentially affect central nervous food intake regulation either via direct access to the brain, vagal afferents or via endocrine messengers, e.g. cholecystokinin (CCK) (Dore et al., 2017).

Although endogenous NUCB2/nesfatin-1 itself regulates feeding behavior, its expression can also be regulated by the feeding state. In rodents, NUCB2 mRNA and NUCB2/nesfatin-1 protein levels were found to be reduced by fasting, and normalized by refeeding, both centrally in the supraoptic nucleus (SON) and hypothalamic paraventricular nucleus (PVN) as well as peripherally in subcutaneous adipose tissue and plasma (Shimizu et al., 2009; Dore et al., 2017). Finally, peripheral and central acute injection of nesfatin-1 midsegment reduced food intake in mice fed a high-fat diet, whereas leptin did not, indicating that long term exposure to high-fat diet did not cause 'nesfatin-1 resistance' analogous to the welldescribed leptin-resistance. Thus, nesfatin-1 could represent a valid alternative in the treatment of metabolic diseases even in the state of leptin resistance (Dore et al., 2017). Subcutaneous administration of nesfatin-1 also inhibited food intake, but its anorexigenic action continued for $14 \mathrm{~h}$ after the injection longer than that by intraperitoneal administration. These results suggested that peripherally administered nesfatin-1 could reduce food intake in mice (Shimizu et al., 2009). After injection into the lateral brain ventricle (intracerebroventricular, icv) of rats, nesfatin-1 reduced dark phase food intake with a delayed onset (maximum 87\% reduction during the third hour post injection) and a long duration of action (lasting 6-48 h). This finding was reproduced in mice with a delayed $(2 \mathrm{~h})$ onset of the anorexigenic effect following icv injection and a duration of $8 \mathrm{~h}$. Interestingly, when injected into the third or fourth brain ventricle or into the cisterna magna, the anorexigenic action of nesfatin- 1 was already observed during the first hour post injection in rats giving rise to a different downstream mediation of nesfatin-1's effect in forebrain versus hindbrain (Stengel, 2015).

Nesfatin-1 injected acutely icv in mice decreased dark phase food intake via a reduction of meal size (indicating an increase of satiation) as well as meal frequency along with a prolongation of inter-meal intervals (as an indicator of increased satiety) (Goebel et al., 2011). In addition, the mid fragment nesfatin$1_{30-59}$, recently identified as the active core of nesfatin-1, injected icv in rats also reduced food intake via an increase of inter-meal intervals, while meal size was not altered. This difference may be related to species differences in mice versus rats or indicate different receptors/receptor areas responding to full length versus mid fragment nesfatin-1 and/or differential downstream signaling leading to the different microstructures observed (Stengel, 2015). 
Nesfatin-1-induced anorexia occurred in Zucker fatty rats with a leptin receptor mutation, and previous injection of anti-nesfatin-1 antibody did not block leptin-induced anorexia, indicating a possible lack of involvement of hypothalamic leptin signaling in the induction of anorexia by nesfatin- 1 . Taking it into a consideration that peripherally administered mid-segment of nesfatin- 1 inhibits food intake in leptinresistant obese mice, exogenously administered nesfatin- 1 is expected to effectively suppress appetite in leptin-resistant condition, such as human obesity (Shimizu et al., 2009).As mentioned, nesfatin-1 exerts its anorexigenic functions in a leptin-independent manner. Rather, nesfatin-1 signaling seems to be important in mediating leptin-induced anorexia. Nesfatin-1 was also shown to alter meal pattern. In mice, central administration of nesfatin- 1 reduced meal size and increased inter-meal intervals indicating both satiety (meal termination) and satiation (delayed meal initiation), whereas central injection of nesfatin-1 midsegment induced satiety without affecting satiation. Interestingly, in rats fed normal chow, central nesfatin-1 midsegment induced satiation, whereas satiety was induced in rats with diet-induced obesity. As suggested by the authors, these mixed results could be explained by differences in species, receptorbinding affinity between full-length nesfatin-1 vs its midsegment and/or the activation of different signaling pathways depending on dietary conditions (Dore et al., 2017). In a recent study, treatment with nesfatin-1 resulted in the upregulation of Cck (a satiation peptide) and in the downregulation of peptide Yy (PYY, a satiety peptide) mRNA and protein levels both in vitro and in vivo (Ramesh et al., 2016).

Nesfatin-1 might be involved not only in regulating homeostatic feeding but also in the reward-related aspects of feeding behavior. In fact, NUCB2/nesfatin-1 protein is expressed in reward-related areas such as amygdaloid nucleus, nucleus accumbens (NAcc), lateral hypothalamus and dorsal raphe nuclei (DR). Nesfatin-1 may decrease the resting membrane potential and firing rate of dopaminergic neurons most likely by a direct mechanism. Dopaminergic neurons originating from the ventral tegmental area (VTA) innervate the NAcc and release dopamine in response to rewarding (or aversive) stimuli. Interestingly, i.c.v. nesfatin-1 decreases dopamine release in the NAcc (Ramesh et al., 2016).

Whether nesfatin-1 acts directly or also recruits other neurotransmitter systems to modulate reward pathways is yet to be elucidated. One possibility is that NUCB2/nesfatin-1 neurons interact with melanocortin and oxytocin systems, which are mediating nesfatinl's effects on homeostatic food intake. In support of this idea, melanocortins and oxytocin were shown to regulate not only homeostatic feeding but also play a role in the modulation of hedonic pathways. A second possible scenario might be the interaction between the nesfatin-1 and ghrelin systems. Ghrelin and nesfatin-1 are co-expressed in X/A endocrine cells of the gastric mucosa. Ghrelin regulates homeostatic feeding by increasing food intake in both rodents and humans. An interaction of nesfatin-1 and ghrelin is also supported by data showing their co-localization in goldfish hypothalamus. Moreover, i.c.v. nesfatin-1 suppresses food intake and downregulates preproghrelin and ghrelin receptor mRNA expression in the forebrain of fed fish. In a similar fashion, i.c.v. ghrelin promotes food intake and downregulates NUCB2 mRNA expression in the forebrain. Accordingly, lipopolysaccharideinduced acute inflammation in rats increased plasma NUCB2/nesfatin-1 levels, whereas those of acyl and desacyl ghrelin were decreased in conjunction with reduced food intake, thus suggesting that NUCB2/nesfatin-1 and ghrelin expression might be regulated differentially (Dore et al., 2017).

In a study, Nesfatin-1 immunoreactivity was identified in the rat gastric mucosa, and most of nesfatin-1 immunoreactive cells co-express ghrelin (Stengel et al., 2009). They raised the possibility that nesfatin/NUCB2 gene expression may be regulated by nutritional status, suggesting a regulatory role of peripheral nesfatin-1 in energy homeostasis. The gastric vagal afferent is the major pathway conveying stomach-derived ghrelin's signal for starvation. It is possible that anorexigenic signal of peripherally administered nesfatin-1 and its active mid-segment may be transported to the brainstem via vagal nerve, similarly with ghrelin (Shimizu et al., 2009). In a study, nesfatin-1 inhibits the appetite of Siberian sturgeon mainly through the CCK-CCK1R signaling pathway (Zhang et al., 2018).

NUCB2/nesfatin-1 was not only described as a direct mediator of central nervous regulation of food intake but also as a regulator of gastrointestinal functions, e.g. by slowing down gastric emptying. Nesfatin-1 appears to contribute to the regulation of food intake by directly and/or indirectly affecting the integration of input and output signals between gut and brain (Dore et al., 2017). As observed for several food intake regulatory peptides before, also NUCB2/nesfatin-1 was implicated in the regulation of gastrointestinal functions. Nesfatin-1 injected into the fourth brain ventricle inhibited the vagally mediated stimulation of gastric acid secretion by 2-deoxy-D-glucose-, whereas the basal or pentagastrin stimulated gastric acid secretion was not altered. In addition, nesfatin-1 was also shown to affect gastrointestinal motility. When injected icv, nesfatin-1 inhibited gastric emptying in rats and mice. In addition, in mice icv nesfatin-1 was also shown to slow down gastroduodenal motility. Conversely, blockade of endogenous NUCB2/nesfatin1 signaling using an anti-NUCB2/nesfatin-1 antibody injected into the ARC or PVN enhanced gastric 
motility giving rise to a physiological effect, which may contribute to the anorexigenic action as gastric distension increases vagal afferent activity. In addition, also NUCB2/nesfatin-1 neurons in the NTS were activated by gastric distention, which may indicate an additional mechanism to further reduce gastrointestinal motility. Lastly, also peripheral NUCB2/nesfatin-1 may contribute to these alterations as rats with a lesion in the ventromedial hypothalamic nucleus showed increased gastric emptying which was associated with higher expression levels of NUCB2 mRNA and NUCB2/nesfatin-1 peptide in the stomach and duodenum (Stengel, 2015).

\section{Nesfatin-1 and obesity}

Nesfatin-1 has an inhibitory effect on food intake and thus alleviates obesity in a dose- and time-dependent manner upon intracerebroventricular and intraperitoneal injection, as well as after intranasal administration (Ozkan et al., 2013). The expression of NUCB2 mRNA in peripheral tissues comprising heart, spinal cord, pancreas, islets, stomach, and adipose tissue, muscle have crucial physiological roles in body weight and they also contribute to the pathophysiology of insulin resistance and its associated metabolic problems in obesity and diabetes (Khalili et al., 2017). There is a slightly positive but inconsistent correlation of nesfatin-1 with body mass index (BMI) in humans, since variables as sex and stress seem to confound this relationship (Ozkan et al., 2013; Prinz et al., 2016).

Plasma levels of nesfatin-1 can correlate with body mass index (Yosten, 2013; Stengel, 2015), insulin resistance, fasting blood glucose, and fasting insulin levels, as well as with body weight and fat mass, suggesting that nesfatin-1, especially fat-derived nesfatin-1, may play an important rol in human metabolism and food intake (Yosten, 2013). In a study, serum nesfatin-1 levels in obese and non-obese subjects were $2.49 \pm 1.96 \mathrm{ng} / \mathrm{mL}$ and $0.70 \pm 0.81 \mathrm{ng} / \mathrm{mL}$, respectively. In the same study, fasting serum nesfatin-1 level positively correlated with body fat \%, fat mass and fat free mass (Anwar et al., 2014). In a study by Abaci et al [(Abaci et al., 2013)], serum nesfatin-1 levels of 37 obese children (BMI above the 95th percentile) and 31 healthy children (BMI below the 85th percentile) were compared. The mean level of nesfatin in obese subjects and control group were $1.53 \pm 1.63 \mathrm{ng} / \mathrm{mL}, 2.66 \pm 1.0 \mathrm{ng} / \mathrm{mL}$, respectively. Serum nesfatin-1 level of the obese subjects was significantly lower than that of the control subjects $(\mathrm{p}=0.001)$. In a study by Mirzaei et al (Mirzaei et al., 2015), a positive correlation was found between body fat $\%$ and nesfatin- 1 level. The percentage of body fat in individuals with low, medium and high levels of nesfatin- 1 levels were $38.17 \pm 1.19 \%, 43.03 \pm 0.89 \%, 41.04 \pm 1.08 \%$, respectively.

Nesfatin-1 could influence energy balance regulation. However, little is known regarding the conditions that could influence its expression and modulation. In humans, nesfatin-1 levels are lower in patients with type 1 or 2 diabetes. On the other hand, the potential associations between nesfatin- 1 levels and a number of metabolic parameters such as body mass index (BMI), body fat, fat-free mass, glucose and insulin concentrations are debated Nesfatin-1 may display glucose-dependent insulinotropic effects. This effect was confirmed by the lowering of blood glucose in hyperglycemic $\mathrm{db} / \mathrm{db}$ mice in response to intravenous administrations of nesfatin-1 (St-Pierre et al., 2016).

Nesfatin-1 might well be involved in long-term changes of energy expenditure. Because nesfatin-1 reduces food intake and increases energy expenditure, it induces a negative energy balance, which might be relevant in states of overnutrition but also might reflect conditions of stress, ultimately leading to wasting or cachexia. With regard to lipid metabolism, chronic subcutaneous infusion of nesfatin-1 may reduce cholesterol and triglyceride levels in mouse plasma, an effect independent of food intake and body weight. Lipid accumulation may be reduced by nesfatin- 1 in cultured hepatocytes in vitro, probably because of a decrease of lipogenesis-relevant genes such as peroxisome proliferator-activated receptor-g and sterolregulatory element-binding protein 1 and enzymes including fatty acid synthase and glycerol-3phosphatase acyltransferase, whereas b-oxidation-related genes may be increased. Because circulating levels of NUCB2/nesfatin-1 may be reduced in patients with nonalcoholic fatty liver disease, the peptide might play a pathogenetic role under these conditions (Schalla et al., 2018).

\section{Nesfatin-1 and glucose homeostasis}

Nesfatin-1 may support insulin sensitivity. Nesfatin-1 treatment can decrease blood glucose level and improve insulin sensitivity (Mogharnasi et al., 2019). Hypothalamic nesfatin-1 action through a neuralmediated pathway can contribute to increased peripheral and hepatic insulin sensitivity by decreasing gluconeogenesis and promoting peripheral glucose uptake in vivo (Yang et al., 2012). In mice, continuous subcutaneous infusion of nesfatin-1 may reduce blood glucose during an oral glucose tolerance test (OGTT). A modulation glucose metabolsm by peripheral rather than central nesfatin-1 (Prinz et al., 2016). In a study by Akın et al. (Akin et al., 2017), fasting plasma nesfatin-1 levels of patients with IGT were higher than the control group. The mean fasting nesfatin-1 levels of patients with IGT and control group 
were $2.1 \pm 2.6 \mathrm{ng} / \mathrm{mL}, 0.5 \pm 0.2 \mathrm{ng} / \mathrm{mL}$, respectively $(\mathrm{p}=0.033)$. In a study conducted by Akour et (Akour et al., 2017), the nesfatin-1 levels of normoglycemic and pre-DM/T2DM individuals were compared. The mean of nesfatin-1 levels of normoglycemic individuals and individuals with pre-DM/T2DM were 0.24 $\mathrm{ng} / \mathrm{mL}$ and $0.50 \mathrm{ng} / \mathrm{mL}$, respectively. Patients with pre-DM and T2DM had higher serum level of nesfatin than normoglycemic individuals $(\mathrm{p}=0.000)$.

The anti-hyperglycemic effect of nesfatin-1 results not only from its endocrine function but also from its inhibition of hepatic glucose production by way of the regulation of glycogen synthesis and gluconeogenesis. The ICV injection of nesfatin regulated hepatic gluconeogenesis to inhibit hepatic glucose production. Nesfatin-1 does this by either decreasing the synthesis of the phosphoenolpyruvate carboxykinase enzyme, which is a rate-limiting enzyme in gluconeogenesis in the liver, or by increasing the activity of the insulin hormone that causes a decrease in the hepatic glucose production (Aydin, 2013). Nesfatin-1 increases the expression of pre-proinsulin mRNA and stimulates the release of insulin induced by glucose via L-type channels (Stengel, 2015). The regulatory role of nesfatin-1 in glucose homeostasis can produce an antihyperglycemic effect. The detection of nesfatin-1 located in beta cell containing insulin could provide an evidence of its connection with glucose metabolism (Algul et al., 2016). In a study by Algül et al (Algul et al., 2016), the nesfatin-1 levels were lower, although not statistically significant, in IFG $(0.937 \pm 0.03 \mathrm{ng} / \mathrm{ml}, \mathrm{p}=0.07)$ and IGT $(1.039 \pm 0.06 \mathrm{ng} / \mathrm{ml}, \mathrm{p}=0.5)$ groups compared to healthy subjects $(1.094 \pm 0.07 \mathrm{ng} / \mathrm{ml})$.

The glucose lowering effect of nesfatin-1 is also exerted in other organs as basal and insülin-stimulated glucose uptake in primary adipocytes treated with nesfatin-1 may be increased. Insulin and subcutaneous infusion of nesfatin-1 may increase protein kinase B (AKT) phosphorylation. (Prinz et al., 2016).

NUCB2 may have a chief role in insulin resistance or T2DM. The beginning of T2DM classically follows a change in glucose metabolism symbolized by insulin resistance, atypical fasting glucose levels, and reduced glucose tolerance. Managing of glucose intolerance is often problematic, because of inadequate findings about the mechanisms by which over-nutrition harms glucose homeostasis (Khalili et al., 2017). Hypoglycemia activates nesfatin expressions in the hypothalamic neurons. It was also reported that elevated glucose in blood increased the release of nesfatin1 in the endocrine cells of pancreas (Aydin, 2013). Additionally, high level of glucose in blood improved the release of nesfatin-1 in the endocrine cells of pancreas. Chronic changes in glucose concentrations influence the pancreatic NUCB2 expression proposing a controlling action in glucose homeostasis, particularly under situations of pathologically changed blood glucose levels (Khalili et al., 2017). The effects of nesfatin-1 on insulin were also observed in vivo as an intravenous injection of nesfatin-1 decreased blood glucose levels in hyperglycaemic $\mathrm{db} / \mathrm{db}$ mice likely due to a stimulated incretin-amplified insulin-induced glucose uptake involving increased GLUT4 membrane translocation in skeletal muscle and adipose tissue The implication of NUCB2/nesfatin-1 in glucose control is also supported by alterations observed in the brain where glucose and insulin activated NUCB2/nesfatin-1 containing neurons of the PVN, while NUCB2/nesfatin-1 positive neurons of the dorsal motor nucleus of the vagus nerve (DMV) may activate by hypoglycemia (Stengel, 2015).

Nesfatin-1 may positively correlate with percentage of calories derived from daily carbonhydrates saturated fat intake, while it may negatively correlate with percentage of calories derived from daily protein intake (Anwar et al., 2014).

\section{Conclusion}

Nesfatin-1 is an 82-amino acid hormone that derived from the nucleobindin-2 (NUCB2) precursor protein. This polypeptide expressed both in the brain and peripheral tissues and involved in the control of energy balance by reducing food intake. Nesfatin-1 may reduce in weight gain and fat depots related to the anorexigenic action. Nesfatin-1 might be involved not only in regulating homeostatic feeding but also in the reward-related aspects of feeding behavior. Nesfatin-1 may decrease the resting membrane potential and firing rate of dopaminergic neurons most likely by a direct mechanism. Nesfatin-1 appears to contribute to the regulation of food intake by directly and/or indirectly affecting the integration of input and output signals between gut and brain. Plasma levels of nesfatin-1 can correlate with body mass index, insulin resistance, fasting blood glucose, and fasting insulin levels, as well as with body weight and fat mass. Nesfatin-1 might well be involved in long-term changes of energy expenditure. Because nesfatin-1 reduces food intake and increases energy expenditure, it induces a negative energy balance. Nesfatin-1 may support insulin sensitivity. Nesfatin-1 treatment can decrease blood glucose level and improve insulin sensitivity. Due to all these effects, nesfatin-1 may be useful in the treatment of obesity, insulin resistance and Type 2 diabetes. Studies on this subject will shed light on the use of nesfatin-1 in treatment. 


\section{References}

Abaci, A., G. Catli, A. Anik, T. Kume and E. Bober (2013). The relation of serum nesfatin-1 level with metabolic and clinical parameters in obese and healthy children. Pediatr Diabetes 14(3): 189-195. doi: 10.1111/pedi.12009

Akın, Ş., N. G. Ersöz, D. Y. Aksoy, J. Karakaya and A. Usman (2017). Increased Serum Nesfatin-1 Levels in Patients with Impaired Glucose Tolerance. Turk J Endocrinol Metab 21: 65-67. doi: 10.25179/tjem.2017-56543.

Akour, A., V. Kasabri, N. Boulatova, Y. Bustanji, R. Naffa, D. Hyasat, N. Khawaja, H. Bustanji, A. Zayed and M. J. A. D. Momani (2017). Levels of metabolic markers in drug-naive prediabetic and type 2 diabetic patients. 54(2): 163-170. doi: 10.1007/s00592-016-0926-1.

Algul, S., Y. Ozkan and O. Ozcelik (2016). Serum nesfatin-1 levels in patients with different glucose tolerance levels. Physiol Res 65(6): 979-985.

Anwar, G. M., G. Yamamah, A. Ibrahim, D. El-Lebedy, T. M. Farid and R. Mahmoud (2014). Nesfatin-1 in childhood and adolescent obesity and its association with food intake, body composition and insulin resistance. Regul Pept 188: 21-24. doi: 10.1016/j.regpep.2013.12.001.

Aydin, S. J. E. (2013). Multi-functional peptide hormone NUCB2/nesfatin-1. J Endocrin 44(2): 312 325. doi: 10.1007/s12020-013-9923-0.

Dore, R., L. Levata, S. Gachkar, O. Johren, J. Mittag, H. Lehnert and C. Schulz (2017). The thermogenic effect of nesfatin-1 requires recruitment of the melanocortin system. $J$ Endocrinol 235(2): 111-122. doi: 10.1530/JOE-17-0151.

Dore, R., L. Levata, H. Lehnert and C. Schulz (2017). Nesfatin-1: functions and physiology of a novel regulatory peptide. J Endocrinol 232(1): R45-R65. doi: 10.1530/JOE-16-0361.

Folgueira, C., L. M. Seoane and F. F. Casanueva (2014). The brain-stomach connection. Front Horm Res 42: 83-92. doi: 10.1159/000358316.

Goebel, M., A. Stengel, L. Wang and Y. Taché (2011). Central nesfatin-1 reduces the nocturnal food intake in mice by reducing meal size and increasing inter-meal intervals. Peptides 32(1): 36-43. doi: 10.1016/j.peptides.2010.09.027.

Khalili, S., M. Shekari Khaniani, F. Afkhami and S. Mansoori Derakhshan (2017). NUCB2/Nesfatin1: A Potent Meal Regulatory Hormone and its Role in Diabetes. Egyptian Journal of Medical Human Genetics 18(2): 105-109. doi: 10.1016/j.ejmhg.2016.10.003.

Mirzaei, K., A. Hossein-nezhad, S. A. Keshavarz, F. Koohdani, M. R. Eshraghian, A. A. SaboorYaraghi, S. Hosseini, M. Chamari, M. Zareei and M. Djalali (2015). Association of nesfatin-1 level with body composition, dietary intake and resting metabolic rate in obese and morbid obese subjects. Diabetes \& Metabolic Syndrome: Clinical Research \& Reviews 9(4): 292-298. doi: 10.1016/j.dsx.2014.04.010.

Mogharnasi, M., H. TaheriChadorneshin, S. A. Papoli-Baravati and A. Teymuri (2019). Effects of upper-body resistance exercise training on serum nesfatin-1 level, insulin resistance, and body composition in obese paraplegic men. Disability and Health Journal 12(1): 29-34. doi: 10.1016/j.dhjo.2018.07.003.

Oh, I. S., H. Shimizu, T. Satoh, S. Okada, S. Adachi, K. Inoue, H. Eguchi, M. Yamamoto, T. Imaki, K. Hashimoto, T. Tsuchiya, T. Monden, K. Horiguchi, M. Yamada and M. Mori (2006). Identification of nesfatin-1 as a satiety molecule in the hypothalamus. Nature 443(7112): 709-712. doi: 10.1038/nature05162. 
Ozkan, Y., E. S. Timurkan, S. Aydin, I. Sahin, M. Timurkan, C. Citil, M. Kalayci, M. Yilmaz, A. Aksoy and Z. Catak (2013). Acylated and desacylated ghrelin, preptin, leptin, and nesfatin-1 Peptide changes related to the body mass index. Int $J$ Endocrinol 2013: 236085. doi: $10.1155 / 2013 / 236085$.

Prinz, P. and A. Stengel (2016). Expression and regulation of peripheral NUCB2/nesfatin-1. Current Opinion in Pharmacology 31: 25-30. doi: 10.1016/j.coph.2016.08.012.

Prinz, P. and A. Stengel (2016). Nesfatin-1: current status as a peripheral hormone and future prospects. Current Opinion in Pharmacology 31: 19-24. doi: 10.1016/j.coph.2016.08.011.

Ramesh, N., S. Mortazavi and S. Unniappan (2016). Nesfatin-1 stimulates cholecystokinin and suppresses peptide YY expression and secretion in mice. Biochem Biophys Res Commun 472(1): 201-208. doi: 10.1016/j.bbrc.2016.02.095.

Schalla, M. A. and A. Stengel (2018). Current Understanding of the Role of Nesfatin-1. J Endocr Soc 2(10): 1188-1206. doi: 10.1210/js.2018-00246.

Shimizu, H., A. Ohsaki, S. Oh-I, S. Okada and M. Mori (2009). A new anorexigenic protein, nesfatin1. Peptides 30(5): 995-998. doi: 10.1016/j.peptides.2009.01.002.

St-Pierre, D. H., J. Martin, H. Shimizu, Y. Tagaya, T. Tsuchiya, S. Marceau, L. Biertho, M. Bastien, S.-M. Caron-Cantin, S. Simard, D. Richard, K. Cianflone and P. Poirier (2016). Association between nesfatin-1 levels and metabolic improvements in severely obese patients who underwent biliopancreatic derivation with duodenal switch. Peptides 86: 6-12. doi: 10.1016/j.peptides.2016.09.014.

Stengel, A. (2015). Nesfatin-1 - More than a food intake regulatory peptide. Peptides 72: 175-183. doi: 10.1016/j.peptides.2015.06.002.

Stengel, A., M. Goebel, I. Yakubov, L. Wang, D. Witcher, T. Coskun, Y. Taché, G. Sachs and N. W. G. Lambrecht (2009). Identification and Characterization of Nesfatin-1 Immunoreactivity in Endocrine Cell Types of the Rat Gastric Oxyntic Mucosa. Endocrinology 150(1): 232-238. doi: 10.1210/en.2008-0747.

Su, Y., J. Zhang, Y. Tang, F. Bi and J. N. Liu (2010). The novel function of nesfatin-1: antihyperglycemia. Biochem Biophys Res Commun 391(1): 1039-1042. doi: 10.1016/j.bbrc.2009.12.014.

Yang, M., Z. Zhang, C. Wang, K. Li, S. Li, G. Boden, L. Li and G. Yang (2012). Nesfatin-1 action in the brain increases insulin sensitivity through Akt/AMPK/TORC2 pathway in diet-induced insulin resistance. Diabetes 61(8): 1959-1968. doi: 10.2337/db11-1755.

Yosten, G. L. C. (2013). Chapter One - Novel Neuropeptides in the Control of Food Intake: Neuronostatin and Nesfatin-1. Vitamins \& Hormones. G. Litwack, Academic Press. 92: 1-25.

Zhang, X., J. Qi, N. Tang, S. Wang, Y. Wu, H. Chen, Z. Tian, B. Wang, D. Chen and Z. Li (2018). Intraperitoneal injection of nesfatin-1 primarily through the CCK-CCK1R signal pathway affects expression of appetite factors to inhibit the food intake of Siberian sturgeon (Acipenser baerii). Peptides 109: 14-22. doi: 10.1016/j.peptides.2018.09.008. 